CARNETS DE Carnets de géographes

GÉOGRAPHES.

$8 \mid 2015$

Géographie(s) de la lenteur

Du projet politique des Lumières aux géographies nationales

France, Prusse et Grande-Bretagne (1780-1860)

Laura Péaud

\title{
OpenEdition
}

Journals

Édition électronique

URL : http://journals.openedition.org/cdg/334

DOI : $10.4000 /$ cdg.334

ISSN : 2107-7266

Éditeur

UMR 245 - CESSMA

Référence électronique

Laura Péaud, « Du projet politique des Lumières aux géographies nationales », Carnets de géographes [En ligne], 8 | 2015, mis en ligne le 01 septembre 2015, consulté le 23 septembre 2020. URL : http:// journals.openedition.org/cdg/334; DOI : https://doi.org/10.4000/cdg.334

Ce document a été généré automatiquement le 23 septembre 2020.

\section{()) $(9$}

La revue Carnets de géographes est mise à disposition selon les termes de la Licence Creative Commons Attribution - Pas d'Utilisation Commerciale - Pas de Modification 4.0 International. 


\section{Du projet politique des Lumières aux géographies nationales}

France, Prusse et Grande-Bretagne (1780-1860)

Laura Péaud

1 Entre 1780 et 1860 en Europe, la géographie se structure peu à peu en champ scientifique et académique indépendant, et particulièrement en France, Prusse et Grande-Bretagne. Au même moment dans ces trois pays européens, des géographes travaillent à ce que leur champ soit enfin considéré comme une science à part entière, au même titre par exemple que l'histoire ou les mathématiques. Ils construisent leur champ à la faveur d'un renouvellement profond de ses principes institutionnels et épistémologiques, selon un processus similaire dans ces trois sphères. Cela passe dans un premier temps par l'édification de lieux dédiés aux savoirs géographiques: des sociétés de géographie, cartothèques et bibliothèques sont créées dans le but d'assurer matériellement une place à la géographique dans le champ scientifique. Dans le même temps, les géographes organisent progressivement les connaissances géographiques selon une exigence de scientificité, dont ils discutent les modalités. Parmi les interrogations qui parcourent leur rang se trouvent notamment la place du terrain, le recours aux statistiques ou encore le rôle de la cartographie. Il s'agit de se mettre d'accord sur une langue géographique commune.

2 C'est ce moment particulier, la période 1780-1860, qui a fait l'objet de ma recherche doctorale. Ce processus de construction à la fois scientifique et disciplinaire est largement analysé en comparant les mises en œuvre en France, Prusse et GrandeBretagne. Mais ce qui m'intéressait également, était de mettre en évidence les liens ambigus, toujours complexes et parfois paradoxaux, que cette entreprise de construction disciplinaire parallèle dans trois sphères entretenait avec le et la politique, au moment où l'idée de nation prend forme en Europe. Par le politique, j'entends les acteurs et les institutions des sphères de pouvoir, et par la politique, ce sont les choix, les enjeux, les stratégies mises en œuvre qui sont concernés.

3 Ce qui m'intéressait au premier chef dans ce moment se situe dans le paradoxe d'une géographie qui se définit alors par son objet universel et son ambition universaliste, 
réfutant a priori toute appartenance nationale mais qui pourtant se construit en fait suivant des cadres nationaux et des manières de faire dictées, ou du moins influencées, par les plus hautes sphères du pouvoir. La problématique suivante a guidé mon travail : en quoi le processus disciplinarisation des savoirs géographiques engagé simultanément en France, en Prusse et en Grande-Bretagne dans la période 1785-1860 se trouve-t-il fondamentalement en tension entre, d'une part, un désir d'universel portée à l'échelle européenne par le champ scientifique et, d'autre part, la nationalisation progressive des savoirs géographiques? L'enjeu principal consistait donc pour moi à élucider comment la montée en discipline progressive des savoirs géographiques se trouve constamment empreinte d'une double influence : à la volonté, dans un esprit humaniste et hérité des Lumières, de construire un champ scientifique autonome et au service du progrès de l'humanité, semble en effet répondre une présence toujours plus forte du politique et, en particulier, de l'échelon national. L'enjeu de cette thèse résidait donc très précisément dans l'identification des modalités d'articulation de ces deux polarités et de leurs effets sur la construction du champ géographique.

4 Le sujet ainsi posé, un des enjeux de ce travail a été de fixer un corpus cohérent. Cela nécessitait de réfléchir tout d'abord aux matériaux mobilisés. L'expérience de mes précédentes recherches m'a donné envie de poursuivre sur une utilisation de matériaux complémentaires, relevant du domaine de l'édité, du public et du domaine du privé. J'ai donc composé un corpus à partir d'une part de matériaux textuels et graphiques au statut public et officiel (c'est-à-dire des livres, des revues, des articles scientifiques, des cartes, des atlas, etc.) et d'autre part de documents relevant du domaine de l'intime ou de l'informel (en particulier les correspondances). Ces sources de deux natures différentes donnent à voir deux échelles, celle du collectif et celle de l'individuel que je me suis donné pour but de faire dialoguer.

5 La mise en œuvre de ce programme de recherche s'est faite d'un point de vue méthodologique sur le principe du croisement : croisement des sphères, des matériaux, mais aussi des matrices théoriques et pratiques, en cherchant à allier les outils des géographes et ceux de l'histoire et de la philosophie des sciences. Je me suis notamment nourrie des approches développées par les cultural studies, permettant de réfléchir les liens entre sphère géographique et sphère politique sur un mode réticulaire et pas seulement vertical. Et c'est en particulier à travers la problématique des savoirs situés que j'ai élaboré mes analyses. Cette approche s'est imposée en histoire des sciences depuis quelques années permet de reconsidérer l'entrée par l'espace, en posant la catégorie du lieu au centre des réflexions. Rapportée à ma recherche, cette problématique m'a permis de questionner la concordance ou la discordance des centralités, mais aussi des réseaux, et d'une manière générale des dispositifs spatiaux géographiques et politiques.

\section{Trois résultats principaux}

6 Tout d'abord, ce travail a d'abord montré que politique, de même que la politique constituent un facteur structurant, et pas seulement encadrant du mouvement de disciplinarisation géographique. Ils interviennent sur trois plans. La fonction opératoire du politique est sans doute le plus clairement marquée sur le plan sociologique et institutionnel: le politique finance, encourage les opérations 
géographiques, est en partie à l'origine de l'institutionnalisation de la discipline. D'un point de vue strictement sociologique, si les cercles politiques et géographiques ne se confondent pas tout à fait, ils se recoupent largement. D'un point de vue épistémologique ensuite, le champ politique influence et oriente les finalités, et donc objets, les méthodes, les pratiques, c'est-à-dire tout l'outillage intellectuel qui préside à la construction de l'édifice disciplinaire. Enfin, d'un point historiographique, les choses sont moins nettement fixées. Si des indices d'une stratégie rhétorique révélant que les savoirs géographiques se trouvent au service du politique apparaissent, cela se fait d'une manière différenciée selon les sphères

7 De plus, l'influence politique opère en se renforçant au cours de la période, mais de manière chronologiquement différenciée. Trois périodes peuvent ainsi être distinguées. Entre 1780 et 1815 : le politique est un acteur montant des balbutiements disciplinaires. C'est notamment en grande partie à travers la géographie militaire (de la Révolution puis de l'Empire) que les savoirs géographiques se structurent en France ou en Prusse. Entre 1815 et 1840 : les géographes prennent à leur tour en charge le mouvement d'institutionnalisation disciplinaire, mais les acteurs politiques y sont aussi présents. La politique de renforcement de l'échelon national s'impose peu à peu et contrecarre les exigences universalistes. Cette politique, spécialement dans sa déclinaison coloniale et colonialiste, devient un facteur déterminant de la période suivante, 1840-1860. Le politique informe alors clairement les savoirs géographiques et leurs modalités de production pour coïncider avec les politiques menées nationalement.

Cette influence du et de la politique se manifeste également par une tendance à la nationalisation des savoirs géographiques. Le moment de fondation disciplinaire se révèle en effet marqué par les cadres nationaux dans lequel il s'inscrit. En dépit des annonces liminaires, celles que l'on retrouve notamment dans les statuts des sociétés de géographie, le fonctionnement des premières institutions dédiées aux savoirs géographiques et la fabrication de ces derniers portent la marque de différenciation nationale. C'est le cas en particulier pour les périmètres d'objets, qui se distinguent de plus en plus au fil de la période par leur tendance à recouvrir les sphères d'intérêt nationales. Des éléments autres que politiques, notamment des facteurs culturels, distinguent des manières de faire française, prussienne ou britannique : cela s'observe par exemple en ce qui concerne le gradient observé dans le recours à la théorisation ou dans l'effet de germanisation du vocabulaire géographique. Tous les éléments analysés concordent à accréditer l'image d'une disciplinarisation géographique nationalement différenciée.

Lien vers la thèse

http://theses.univ-lyon2.fr/documents/lyon2/2014/peaud_1

Discipline

Géographie

Directrice

Pr Isabelle Lefort, Université Lumière Lyon 2 - UMR 5600 EVS

Université

Université Lumière Lyon 2 
Membres du jury de thèse, soutenue le 17 novembre 2014

Henri Desbois, Maître de conférences HDR à l'Université Paris Ouest Nanterre la Défense (rapporteur)

Isabelle Lefort, Professeur de géographie à l'Université Lyon 2 (directrice)

Ulrich Päßler, wissenschaftlicher Mitarbeiter à l'Université Humboldt de Berlin Philippe Pelletier, Professeur de géographie à l'Université Lumière Lyon 2 (président)

Jean-Yves Puyo, Professeur de géographie à l'Université de Pau et des Pays de l'Adour (rapporteur)

Situation professionnelle à l'issue de la thèse

ATER à l'Université de Bretagne Sud (Lorient)

Contact de l'auteur

laura.peaud[at]gmail.com

INDEX

Thèmes : Carnets de soutenances 